Article

\title{
Energy-Efficient and Reliable Internet of Things for 5G: A Framework for Interference Control
}

\author{
Radwa Ahmed Osman (D) and Amira I. Zaki *(D) \\ College of Engineering and Technology, Arab Academy for Science, Technology and Maritime Transport, \\ Alexandria 1029, Egypt; radwa.ahmed@aast.edu \\ * Correspondence: amzak10@aast.edu; Tel.: +20-100-661-9608
}

Received: 21 November 2020; Accepted: 15 December 2020; Published: 17 December 2020

\begin{abstract}
The Internet of Things (IoT) is one of the promising paradigms that enable massive machines and devices to communicate with each other in future communication networks to promote a high level of awareness about our world and improve our daily life. IoT devices (IoTDs) communicate with an IoT base station (IoTBS) or IoT gateway (IoTG) by sharing the resources of other cellular users (CUEs). Due to the leakage of the spectral efficiency, interference exists among IoTG and base station (BS) due to CUEs and IoTDs. In this paper, a new framework is proposed called the interference control model. This proposed model aims to control the interference among IoTG and BS and is based on using the Lagrange optimization technique to reduce interference and maximize the energy efficiency and reliability of the IoT and cellular networks in fifth-generation (5G) systems. First, we formulate the multi-objective optimization problem to achieve the objective of the proposed model. Then, based on the optimization strategy, we derive the closed-form expressions of key quality-of-service (QoS) performance such as system reliability, throughput, and energy efficiency. Finally, the proposed algorithm has been evaluated and examined through different assumptions and several simulation scenarios. The obtained results validate the effectiveness and the accuracy of our proposed idea and also indicate significant improvement in the network performance of IoT and cellular networks.
\end{abstract}

Keywords: Internet of Things; 5G; reliability; throughput; energy efficiency; quality of service; optimization

\section{Introduction}

With the rapid development of the Internet of Things (IoT), where all the physical and smart things such as machines, vehicles, and devices are connected through wireless networks to exchange information about themselves and their surroundings, it is a challenge to fulfil user requirements with the required system performance. IoT can enhance our life, improve our daily experience, and cooperate to support various industrial applications by creating an efficient platform for connecting all devices [1,2]. The future of IoT is a bright one because a large amount of IoT devices can communicate and merge to enhance our daily life. For example, the nest smart home-this is an application provided for users on their phones to monitor their devices at their home. This application can provide the user with the optimum required security and convenience. Additionally, the IoT system supports traffic management and vehicle behaviors, and it also enables vehicle-to-vehicle and vehicle-to-person communication to improve transportation safety and traffic management. Moreover, in factories and farms, instruments can communicate and collaborate to enhance the performance of the farms and factories' operations. Many other IoT application scenarios improve and facilitate our daily life [3].

Access networks are responsible for connecting and merging all IoT devices to a centralized infrastructure or access point using either wireless or wired links [4]. Wired links are not suitable for all IoT applications, especially those deployed in remote locations and vehicular applications due to cost 
ineffectiveness and lack of scalability or mobility. On the other hand, they can provide high reliability, high rates, and short delay. Wireless access technologies can either be using capillary networks, which are local networks that use short-range radio-access technologies to provide local connectivity (e.g., wireless local area networks, Zigbee, Wi-Fi, Bluetooth, etc.) [5], or using cellular technology such as the global system for mobile communication (2G/GSM), universal mobile telecommunications system (3G/UMTS), Long-Term Evolution (4G/LTE), fifth-generation (5G) mobile network, etc. The wireless capillary is considered as a low-power and lossy network [6]; additionally, it is relatively low cost and scalable. However, cellular networks are considered reliable and support mobility, which makes cellular IoT a promising technology for several applications [7]. Moreover, the evolving fifth-generation (5G) cellular wireless networks provide higher data rates, enhance end-user quality of experience (QoE), reduce end-to-end latency, and lower energy consumption [8]. Furthermore, 5G networks are expected to massively expand today's IoT, which can boost cellular operations, IoT security, and network challenges and drive the Internet future to the edge [9].

Fifth-generation (5G) technologies enable machine-to-machine (M2M), device-to-device (D2D), and device-to-everything (D2E) communication, IoT, and Internet of Vehicles (IoV). This kind of communication will require an enormous demand in future communication systems, which will be fast and will include more connected devices that are normally supported in combined networks. In this work, we have multiple IoT devices and cellular users (CUEs) sharing the same spectrum, transmitting and receiving data to IoT gateways and base stations (BSs), respectively. We investigate the effect of the interference occurring due to the number of transmitting IoT devices (IoTDs) on base stations (BSs) and the number of cellular users (CUEs) on the IoT gateway (IoTG) and find an adequate solution to decrease interference using an optimization strategy to enhance the IoT and cellular network performance in terms of reliability and efficiency under different environmental conditions. These findings are used to identify, based on different constraints and requirements, the suitable and acceptable distance between CUE and IoTG and IoTD and BS that can decrease interference and optimize system performance for the whole network in a changing environment. The proposed approach is unique in the sense that it provides an efficient way to find the most adequate distance for CUE-IoTG and IoTD-BS. This method is evaluated based on the performance model we derive and its simulation. The remainder of this paper is organized as follows. Section 2 discusses the relevance of this research with other work reported. The system model and the problem formulation are presented in Section 3. Simulation results produced by MATLAB and discussions are presented in Section 4. Finally, the paper is concluded in Section 5.

\section{Related Work}

IoT devices' deployment over cellular networks has major technical challenges at the architecture, operational, and economic levels $[10,11]$. Furthermore, satisfying the IoT application requirements along with their associated communication technologies [12] and selecting an efficient service for optimal management of both energy and quality of service (QoS) $[13,14]$ are other challenges to be considered. Thus, different techniques and methods have been studied extensively to show how to enhance the QoS, the communication between IoT devices, and increase the energy efficiency [15]. To meet the requirements of high energy efficiency and large system capacity for the fifth-generation Internet of Things (IoT), the authors of [16] considered the energy-efficient design of a multipair decode-and-forward relay-based IoT network to obtain an accurate and efficient system performance. Moreover, to enhance the transmission efficiency as well as the reception reliability, cooperative relaying to the IoT network was introduced in [17] and a cooperative IoT protocol was proposed. Additionally, for IoT system enhancement, ref. [18] presented multiband cooperative spectrum sensing and a resource allocation framework for IoT in cognitive 5G networks to enhance the system QoS in terms of data rate, latency, reliability, economic price, and environmental cost. On the other hand, ref. [19] defined effective throughput and effective amount of information to enhance transmission efficiency and reliability. 
Concerning the energy consumption for IoT, different techniques and methods have been presented to solve this issue-for example, ref. [20] proposed a power measurement utility for reinforcement learning (PMU-RL) algorithm to dynamically adjust the resource utilization of heterogeneous platforms in order to minimize power consumption, and [21] described an energy-centric and context-aware optimization framework that accounts for the energy impact of the fundamental functionalities of an IoT system. Moreover, a new architecture has been presented in [22] to allocate the IoT network resource and minimize the overall energy consumption of the pico relay base stations. On the other hand, the problem of how to achieve a tradeoff between the QoS provisioning and the energy efficiency for the industrial IoT systems was proposed in [23], to achieve the objective of balancing the outage performance and the network lifetime. Furthermore, an energy- and spectrum-efficient IoT network for 5G systems was presented in [24] to increase the IoT network utilization. Furthermore, it has been proven that the energy consumption of a network can be minimized via joint power control and time allocation [25] or via joint transmission time and power assignment [26]. Additionally, the paper [27] addressed the optimized scheduling of the monitoring role between the embedded devices in IoT networks, to minimize energy consumption and communication overhead of monitoring, for each node. Furthermore, an efficient suboptimal algorithm was proposed [28] to solve the energy efficiency maximization problem and achieve better energy efficiency performance for IoT devices. Moreover, increasing the energy efficiency fairness among heterogeneous mobile devices in interference channels will become a crucial issue in future wireless networks that can be solved using the deep learning approach [29]. However, an elastic cell-zooming algorithm based on the quality of service and traffic loads of end-users was proposed in [30], performed by adaptively adjusting the transmission power of small cells in order to reduce energy consumption for IoT.

Moreover, decreasing the energy consumption for decreasing interference in IoT network is a key issue, depending on the connectivity between IoT devices. Nash non-cooperative power game was an effective method implemented in [31] to decrease the interference between IoT users which largely enhanced the edge IoT user throughput. The sum rate of the IoT cellular network can be maximized by a joint sub-band assignment and power allocation optimization [32] and low-complexity, low-cost IoT communication is enabled by applying a multi-rate filtered orthogonal frequency division multiplexing system (F-OFDM) [33]. Additionally, the mitigation of the IoT interference to LTE-A is an important issue presented in [34] to improve accuracy of and eliminate IoT interference in LTE-A systems.

Although there have been many reports establishing the possibility of improving the system performance of IoT networks by using different proper communication protocols or data processing algorithms, there is a lack of information concerning how to identify the suitable approximate distance between CUE-IoTG and IoTD-BS under different conditions that can decrease the interference and ensure the best system performance for both IoT and cellular networks. In this paper, the main concern is the identification of the conditions for establishing the minimum appropriate distance between CUE-IoTG and IoTD-BS to decrease interference at the BS and IoTG. The proposed approach is based on the development of analytical and simulation models for the proposed approach and the assessment of its performance in reliability, throughput, and energy efficiency on both networks. The suggested approach shows how to achieve optimum performance by adapting the distance between CUE-IoTG and IoTD-BS to enhance their system performance. However, the following Table 1 gives a summary of the comparison between the relevant research literature and the proposed model in this work. 
Table 1. Comparison between the different methods and algorithms presented previously and the current proposed model.

\begin{tabular}{ccccccc}
\hline & $\begin{array}{c}\text { Throughput } \\
\text { Maximization }\end{array}$ & Fairness & Reliability & $\begin{array}{c}\text { Energy } \\
\text { Efficiency }\end{array}$ & $\begin{array}{c}\text { QoS } \\
\text { Satisfaction }\end{array}$ & Utilization \\
\hline $\begin{array}{c}\text { Proposed model } \\
{[14]}\end{array}$ & $\sqrt{ }$ & & $\sqrt{ }$ & $\sqrt{ }$ & $\sqrt{ }$ & \\
{$[15]$} & & $\sqrt{ }$ & & $\sqrt{ }$ & $\sqrt{ }$ & \\
{$[16]$} & $\sqrt{ }$ & & & $\sqrt{ }$ & $\sqrt{ }$ & \\
{$[17]$} & $\sqrt{ }$ & & $\sqrt{ }$ & $\sqrt{ }$ & $\sqrt{ }$ & \\
{$[18]$} & $\sqrt{19]}$ & & $\sqrt{ }$ & & \\
{$[20]$} & $\sqrt{ }$ & & & $\sqrt{ }$ & & $\sqrt{ }$ \\
{$[21]$} & & & $\sqrt{ }$ & $\sqrt{ }$ & $\sqrt{ }$ \\
{$[22]$} & & & $\sqrt{ }$ & & \\
{$[23]$} & $\sqrt{28]}$ & & & $\sqrt{ }$ & & \\
{$[29]$} & $\sqrt{ }$ & & & & &
\end{tabular}

\section{System Model and Problem Formulation}

The analytical models of the suitable distance between IoTD-BS and CUE-IoTG, system outage probability, energy efficiency, and throughput in the context of IoT and cellular networks are established in this section. Based on this model, the interference between IoTD-BS and CUE-IoTG can be controlled and decreased to optimize and enhance the system performance for both networks. Given a heterogeneous cellular network consisting of N CUEs connected to the BS at the center and a cluster of $K$ IoTDs connected to the IoTG, for any CUE-BS, the goal of the optimization proposed in this work is achieved by maximizing energy efficiency $(E E)$, maximizing the end-to-end throughput $\left(S_{t h}\right)$, and minimizing the system outage probability $\left(P_{\text {out }}\right)$ based on controlling the interference between CUE-IoTG and IoTD-BS; i.e.,:

$$
\begin{gathered}
\operatorname{Min} P_{\text {outij }} P_{\text {outij }}:=f_{1}\left(I_{I B}, I_{C G}\right) \text { s.t. }\left\{I_{I B} \leq I_{I B \max }, I_{C G} \leq I_{C G \max }\right\} \text { and } \\
\operatorname{Max} \sum_{i=1, j=K}^{i=1, j=1} E E_{i j} E_{i j}:=f_{2}\left(I_{I B}, I_{C G}\right) \text { s.t. }\left\{I_{I B} \leq I_{I B \max }, I_{C G} \leq I_{C G \max }\right\} \text { and } \\
\operatorname{Max} \sum_{i=N, j=K}^{i=N, j=K} S_{\text {thij }} S_{\text {thij }}:=f_{3}\left(I_{I B}, I_{C G}\right) \text { s.t. }\left\{I_{I B} \leq I_{I B \max }, I_{C G} \leq I_{C G \max }\right\}
\end{gathered}
$$

where $P_{\text {outij }}, E E_{i j}$, and $S_{\text {thij }}$ are the total system outage probability, the total energy efficiency, and the overall system throughput, respectively, of the $i$-th path between a CUE-BS and the $j$-th path between IoTD and IoTG; $I_{I B}, I_{C G}, I_{I B \max }$, and $I_{C G \max }$ are the interference and the maximum acceptable interference between IoTD and BS and CUE and IoTG, respectively.

The transmission scheme in the context of cellular and IoT networks is identified in Figure 1, including direct CUE-BS and direct IoTD-IoTG. The IoTG acts as the data condenser of all the IoTDs and it sends data to the BS by using the available radio resources from the CUEs. In this work, we intend to examine and compare cellular and IoT networks' performance in energy efficiency $(E E)$, throughput $\left(S_{\text {th }}\right)$, and system outage probability $\left(P_{\text {out }}\right)$ and to optimize both network performance in different environmental conditions. 


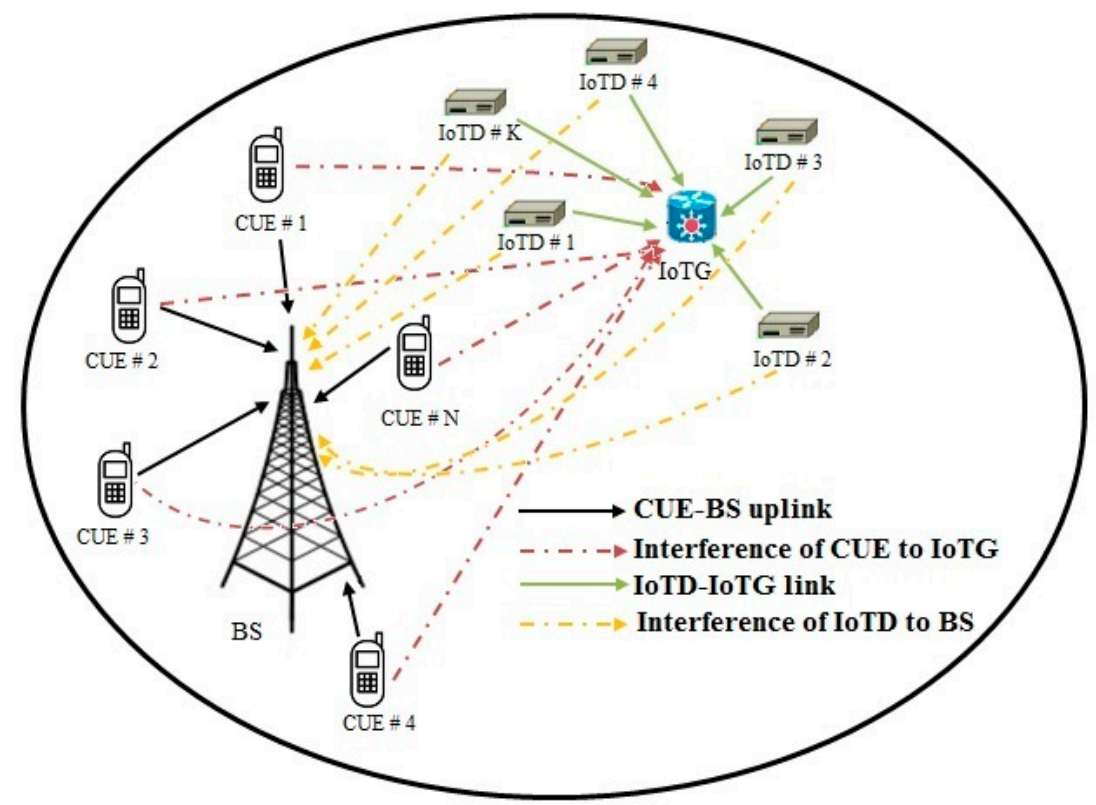

Figure 1. System model.

A narrowband Rayleigh fading with additive white Gaussian noise (AWGN) $[19,35,36]$ and resulting propagation path loss are assumed in the considered system. The channel fading coefficient is assumed to be statistically mutually independent for different links. The signals received at the BS and the IoTG due to the transmissions are given by [35]:

$$
\begin{aligned}
& r_{C B}=\sqrt{P_{C} d_{C B}^{-\alpha}} h_{C B} X+I_{I B}+n_{C B} \\
& r_{I G}=\sqrt{P_{I} d_{I G}^{-\alpha}} h_{I G} Y+I_{C G}+n_{I G}
\end{aligned}
$$

where $P_{C}, d_{C B}$, and $h_{C B}$ are the transmission power, transmission distance, and the channel coefficient, respectively, of the CUE-BS link; $P_{I}, d_{I G}$, and $h_{I G}$ are the transmission power, transmission distance, and the channel coefficient, respectively, of the IoTD-IoTG link; $\alpha$ is the path loss exponent; $X$ and $Y$ are the transmitted symbols of CUE-BS and IoTD-IoTG links, respectively, with unit power; $n_{C B}$ and $n_{I G}$ are the independent and identically distributed (i.i.d.) additive white Gaussian noise (AWGN) of CUE-BS and IoTD-IoTG links, respectively, with variance $N_{o} / 2$ per dimension, where $N_{o}$ is the thermal noise power spectral density per Hertz. The corresponding signal-to-interference ratio (SINR) at the BS and the IoTG are represented by:

$$
\begin{gathered}
\operatorname{SINR}_{C B}=\frac{P_{C}\left|h_{C B}\right|^{2} \gamma_{C B}}{I_{I B}+N_{o} B} \\
\operatorname{SINR}_{I G}=\frac{P_{I}\left|h_{I G}\right|^{2} \gamma_{I G}}{I_{C G}+N_{o} B}
\end{gathered}
$$

where $B$ is the system bandwidth in Hertz; $\gamma_{C B}$ and $\gamma_{I G}$ are the path loss model of CUE-BS and IoTD-IoTG links, respectively. The path loss model between any two nodes $i$ and $j$ at a distance $d i j$ can be expressed as [35]:

$$
\gamma_{i j}=\gamma_{o} d_{i j}{ }^{-\alpha}
$$

where $\gamma_{o}$ is the path loss constant. The interference between IoTD and BS $\left(I_{I B}\right)$ and CUE and IoTG $\left(I_{C G}\right)$ can be given by:

$$
\begin{gathered}
I_{I B}=P_{I}\left|h_{I B}\right|^{2} \gamma_{I B} \\
I_{C G}=P_{C}\left|h_{C G}\right|^{2} \gamma_{C G}
\end{gathered}
$$


where $h_{I B}$ and $h_{C G}$ are the channel coefficients of IoTD-BS and CUE-IoTG links, respectively; $\gamma_{I B}$ and $\gamma_{C G}$ represent the path loss model of IoTD-BS and CUE-IoTG links, respectively. We assume that both destinations have certain QoS constraints that need to be satisfied to correctly decode the received signals from the CUE and the IoTD. The QoS constraints can be satisfied if, and only if, $S I N R_{C B} \geq S I N R_{t h C B}$ and $S I N R_{I G} \geq S I N R_{t h I G}$, where SINR $R_{t h C B}$ and SINR $R_{t h I G}$ are the threshold SINR values at the CUE-BS and the IoTD-IoTG links, respectively. Based on this assumption, IBmax $_{\text {max }}$ and $I_{C G \max }$ can be expressed as:

$$
\begin{aligned}
I_{I B \max } & =\frac{P_{C}\left|h_{C B}\right|^{2} \gamma_{C B}-S_{I N R t h C B} N_{o} B}{S_{I N R t h C B}} \\
I_{C G \max } & =\frac{P_{I}\left|h_{I G}\right|^{2} \gamma_{I G}-S_{I N R t h I G} N_{o} B}{S_{I N R \ln I G}}
\end{aligned}
$$

\subsection{Minimization of Outage Probability under the Interference Control Model}

An outage probability (Pout) is defined as the probability that the SINR at the receiver falls below the required threshold $\beta$ for CUE-BS links and $\eta$ for IoTD-IoTG links and which allows error-free decoding. The outage probability of the single-hop transmission is given by $[35,36]$.

$$
\begin{gathered}
P_{\text {out } C B}=p\left(\operatorname{SINR}_{C B} \leq \beta\right)=1-\frac{P_{C}\left|h_{C B}\right|^{2} \gamma_{C B}}{\beta I_{I B}+P_{C}\left|h_{C B}\right|^{2} \gamma_{C B}}\left(e^{-\left(\frac{\beta N_{o} B}{P_{C}\left|h_{C B}\right|^{2} \gamma_{C B}}\right)}\right) \\
P_{\text {outIG }}=p\left(\operatorname{SINR}_{I G} \leq \eta\right)=1-\frac{P_{I}\left|h_{I G}\right|^{2} \gamma_{I G}}{\eta I_{C G}+P_{I}\left|h_{I G}\right|^{2} \gamma_{I G}}\left(e^{-\left(\frac{\eta N_{o} B}{P_{I}\left|h_{I G}\right|^{2} \gamma_{I G}}\right)}\right)
\end{gathered}
$$

Assuming $\beta N o B<<P_{C}\left|h_{C B}\right|^{2} \gamma_{C B}$ and $\eta N o B<<P_{I}\left|h_{I G}\right|^{2} \gamma_{I G}$, Equations (11) and (12) can be written as:

$$
\begin{gathered}
P_{\text {out } C B}=1-\frac{P_{C}\left|h_{C B}\right|^{2} \gamma_{C B}}{\beta I_{I B}+P_{C}\left|h_{C B}\right|^{2} \gamma_{C B}} \\
P_{\text {outIG }}=1-\frac{P_{I}\left|h_{I G}\right|^{2} \gamma_{I G}}{\eta I_{C G}+P_{I}\left|h_{I G}\right|^{2} \gamma_{I G}}
\end{gathered}
$$

Then, the overall system outage probability can be expressed as:

$$
\begin{gathered}
p_{\text {out }}=p_{\text {out } C B}+p_{\text {outIG }}+p_{\text {out } C B^{*}} p_{\text {outIG }} \\
P_{\text {out }}=1-\frac{\left(P_{C}\left|h_{C B}\right|^{2} \gamma_{C B}\right)\left(P_{I}\left|h_{I G}\right|^{2} \gamma_{I G}\right)}{\left(\beta I_{I B}+P_{C}\left|h_{C B}\right|^{2} \gamma_{C B}\right)\left(\eta I_{C G}+P_{I}\left|h_{I G}\right|^{2} \gamma_{I G}\right)}
\end{gathered}
$$

The main objective of the performance optimization of the proposed model is to minimize the total overall system outage probability under different environmental conditions.

$$
\begin{gathered}
\text { Min } P_{\text {outij }} P_{\text {outiji }}:=f_{1}\left(I_{I B}, I_{C G}\right) \\
\text { s.t. } C 1\left\{I_{I B} \leq I_{I B \max }\right\} \\
\text { C2 } 2\left\{I_{C G} \leq I_{C G \max }\right\}
\end{gathered}
$$

In the formulated optimization problem, $\mathrm{C} 1$ is the constraint that the interference between IoTD and BS $\left(I_{I B}\right)$ must be lower than the maximum acceptable interference between IoTD and BS $\left(I_{I B m a x}\right)$. C2 denotes the constraints that the interference between CUE and IoTG $\left(I_{C G}\right)$ must be lower than the maximum acceptable interference between CUE and IoTG $\left(I_{C G \max }\right)$. To find the solution to the 
optimization problem specified in (17), we now investigate the first-order optimality conditions. The Lagrangian of the optimization problem can be calculated as:

$$
L\left\{d_{I B}, d_{C G}, \lambda_{I B}, \lambda_{C G}\right\}=p_{\text {out }}+\lambda_{I B}\left(I_{I B \max }-I_{I B}\right)+\lambda_{C G}\left(I_{C G \max }-I_{C G}\right) .
$$

where $\lambda_{I B}$ and $\lambda_{C G}$ consider the non-negative Lagrangian multipliers for $\mathrm{C} 1$ and $\mathrm{C} 2$, respectively. By taking the derivative of Equation (18) with respect to $d_{I B}$ and $d_{C G}$, we find the optimal solution to (17) as:

$$
\frac{\partial L\left\{d_{I B}, d_{C G}, \lambda_{I B}, \lambda_{C G}\right\}}{\partial d_{I B}}=0
$$

Then,

$$
\lambda_{I B}=\frac{\beta\left(P_{C}\left|h_{C B}\right|^{2} \gamma_{C B}\right)\left(P_{I}\left|h_{I G}\right|^{2} \gamma_{I G}\right)}{\left(\beta I_{I B}+P_{C}\left|h_{C B}\right|^{2} \gamma_{C B}\right)^{2}\left(\eta I_{C G}+P_{I}\left|h_{I G}\right|^{2} \gamma_{I G}\right)}
$$

Equation (19) shows the value of $\lambda_{I B}$ that satisfies the constraint of the optimization problem.

$$
\frac{\partial L\left\{d_{I B}, d_{C G}, \lambda_{I B}, \lambda_{C G}\right\}}{\partial d_{C G}}=0
$$

Then,

$$
\lambda_{C G}=\frac{\eta\left(P_{C}\left|h_{C B}\right|^{2} \gamma_{C B}\right)\left(P_{I}\left|h_{I G}\right|^{2} \gamma_{I G}\right)}{\left(\beta I_{I B}+P_{C}\left|h_{C B}\right|^{2} \gamma_{C B}\right)\left(\eta I_{C G}+P_{I}\left|h_{I G}\right|^{2} \gamma_{I G}\right)^{2}}
$$

Equation (20) shows the value of $\lambda_{C G}$ that satisfies the constraint of the optimization problem. By taking the derivative of (18) with respect to $\lambda_{I B}$ and $\lambda_{C G}$, we find that:

$$
\begin{aligned}
& \frac{\partial L\left\{d_{I B}, d_{C G}, \lambda_{I B}, \lambda_{C G}\right\}}{\partial \lambda_{I B}}=0 \\
& \frac{\partial L\left\{d_{I B}, d_{C G}, \lambda_{I B}, \lambda_{C G}\right\}}{\partial \lambda_{C G}}=0
\end{aligned}
$$

Then, from the first-order optimality conditions, we can derive the following two propositions. Proposition 1: this defines the optimal required distance between IoTD and BS, and Proposition 2, which defines the optimal required distance between CUE and IoTG. The two propositions aim to decrease the interference and enhance the system performance through maximizing QoS. Thus, to achieve the system model goal, IoTD should be at a distance equal to $d_{I B}$ from the BS and CUE should be at a distance equal to $d_{C G}$ from the IoTG, which is given by:

$$
\begin{aligned}
& d_{I B}=\left(\frac{P_{I}\left|h_{I B}\right|^{2} \gamma_{o} S_{\text {INRth } C B}}{P_{C}\left|h_{C B}\right|^{2} \gamma_{C B}-S_{I N R t h C B} N_{o} B}\right)^{\frac{1}{\alpha}} \\
& d_{C G}=\left(\frac{P_{C}\left|h_{C G}\right|^{2} \gamma_{o} S_{\text {INRthIG }}}{P_{I}\left|h_{I G}\right|^{2} \gamma_{I G}-S_{\text {INRthIG }} N_{o} B}\right)^{\frac{1}{\alpha}}
\end{aligned}
$$

\subsection{Maximization of Energy Efficiency under the Interference Control Model}

Energy efficiency $(E E)$ can be defined as the ratio between the overall system throughput and the total transmission power. $E E$ for the proposed model can be formulated as:

$$
E E=\frac{R_{C B}}{P_{C}+P_{o}}+\frac{R_{I G}}{P_{I}+P_{o}}
$$


where $R_{C B}$ and $R_{I G}$ are the throughput of CUE-BS and IoTD-IoTG links, respectively; $P_{o}$ is the internal circuitry power consumption of the CUE-BS and IoTD-IoTG links. $R_{C B}$ and $R_{I G}$ can be given by:

$$
\begin{gathered}
R_{C B}=B \log _{2}\left(1+\frac{P_{C}\left|h_{C B}\right|^{2} \gamma_{C B}}{I_{I B}+N_{o} B}\right) \\
R_{I G}=B \log _{2}\left(1+\frac{P_{I}\left|h_{I G}\right|^{2} \gamma_{I G}}{I_{C G}+N_{o} B}\right)
\end{gathered}
$$

Then, EE can be expressed as:

$$
E E=\frac{B \log _{2}\left(1+\frac{P_{C}\left|h_{C B}\right|^{2} \gamma_{C B}}{I_{I B}+N_{o} B}\right)}{P_{C}+P_{o}}+\frac{B \log _{2}\left(1+\frac{P_{I}\left|h_{I G}\right|^{2} \gamma_{I G}}{I_{C G}+N_{o} B}\right)}{P_{I}+P_{o}}
$$

We can formulate the EE maximization problem by specifying a maximum interference level in a similar way to the method expressed in (17), which can be stated as:

$$
\begin{gathered}
\operatorname{Max} \sum_{i=1, j=1}^{i=N, j=K} E E_{i j} E_{i j}:=f_{2}\left(I_{I B}, I_{C G}\right) \\
\text { s.t. } C 1\left\{I_{I B} \leq I_{I B \max }\right\} \\
\quad \text { C2 }\left\{I_{C G} \leq I_{C G \max }\right\}
\end{gathered}
$$

To find the solution to the optimization problem specified in (27), we now investigate the first-order optimality conditions. The Lagrangian of the optimization problem can be calculated as:

$$
L\left\{d_{I B}, d_{C G}, \lambda_{I B}, \lambda_{C G}\right\}=E E+\xi_{I B}\left(I_{I B \max }-I_{I B}\right)+\xi_{C G}\left(I_{C G \max }-I_{C G}\right) .
$$

where $\xi_{I B}$ and $\xi_{C G}$ consider the non-negative Lagrangian multipliers for $\mathrm{C} 1$ and $\mathrm{C} 2$, respectively. By taking the derivative of Equation (28) with respect to $d_{I B}$ and $d_{C G}$, we find the optimal solution to (27) as:

$$
\frac{\partial L\left\{d_{I B}, d_{C G}, \xi_{I B}, \xi_{C G}\right\}}{\partial d_{I B}}=0
$$

Therefore,

$$
\xi_{I B}=\frac{1}{\left(P_{C}+P_{o}\right)} \frac{P_{C}\left|h_{C B}\right|^{2} \gamma_{C B}}{\left(I_{I B}+N_{o} B+P_{C}\left|h_{C B}\right|^{2} \gamma_{C B}\right)\left(I_{I B}+N_{o} B\right)}
$$

and

Therefore,

$$
\frac{\partial L\left\{d_{I B}, d_{C G}, \xi_{I B}, \xi_{C G}\right\}}{\partial d_{C G}}=0
$$

$$
\xi_{C G}=\frac{1}{\left(P_{C}+P_{o}\right)} \frac{P_{I}\left|h_{I G}\right|^{2} \gamma_{I G}}{\left(I_{C G}+N_{o} B+P_{I}\left|h_{I G}\right|^{2} \gamma_{I G}\right)\left(I_{C G}+N_{o} B\right)}
$$

Equations (29) and (30) show the values of $\xi_{I B}$ and $\xi_{C G}$ that satisfy the constraint of the optimization problem, respectively. By taking the derivative of (28) with respect to $\xi_{I B}$ and $\xi_{C G}$, we find that:

$$
\frac{\partial L\left\{d_{I B}, d_{C G}, \xi_{I B}, \xi_{C G}\right\}}{\partial \xi_{I B}}=0
$$

Therefore,

$$
d_{I B}=\left(\frac{P_{I}\left|h_{I B}\right|^{2} \gamma_{o} S_{I N R t h C B}}{P_{C}\left|h_{C B}\right|^{2} \gamma_{C B}-S_{I N R t h C B} N_{o} B}\right)^{\frac{1}{\alpha}}
$$


and

$$
\frac{\partial L\left\{d_{I B}, d_{C G}, \xi_{I B}, \xi_{C G}\right\}}{\partial \xi_{C G}}=0
$$

Therefore,

$$
d_{C G}=\left(\frac{P_{C}\left|h_{C G}\right|^{2} \gamma_{o} S_{\text {INRthIG }}}{P_{I}\left|h_{I G}\right|^{2} \gamma_{I G}-S_{\text {INRthIG }} N_{o} B}\right)^{\frac{1}{\alpha}}
$$

where $d_{I B}$ and $d_{C G}$ represent the optimum required distances between IoTD and BS and CUE and IoTG, respectively, to reduce the interference and enhance the system performance in terms of energy efficiency.

\subsection{Maximization of Overall System Throughput under the Interference Control Model}

The overall system throughput can be defined as the sum of the data rates that are delivered to all terminals in a network. The throughput is measured in bit/s or bps. The overall system throughput of the proposed model can be stated as:

$$
\begin{gathered}
S_{t h}=R_{C B}+R_{I G} \\
S_{t h}=B \log _{2}\left(1+\frac{P_{C}\left|h_{C B}\right|^{2} \gamma_{C B}}{I_{I B}+N_{o} B}\right)+B \log _{2}\left(1+\frac{P_{I}\left|h_{I G}\right|^{2} \gamma_{I G}}{I_{C G}+N_{o} B}\right)
\end{gathered}
$$

In a similar way to the method expressed in (17), we can express the overall system throughput maximization problem by:

$$
\begin{gathered}
\operatorname{Max} \sum_{i=1, j=1}^{i=N, j=K} S_{\text {thij }} S_{\text {thij }}:=f_{3}\left(I_{I B}, I_{C G}\right) \\
\text { s.t. } C 1\left\{I_{I B} \leq I_{I B \max }\right\} \\
\text { C2 }\left\{I_{C G} \leq I_{C G \max }\right\}
\end{gathered}
$$

To solve the optimization problem specified in (27), we will investigate the first-order optimality conditions. The Lagrangian of this optimization problem can be found as:

$$
L\left\{d_{I B}, d_{C G}, \Omega_{I B}, \Omega_{C G}\right\}=S_{t h}+\Omega_{I B}\left(I_{I B \max }-I_{I B}\right)+\Omega_{C G}\left(I_{C G \max }-I_{C G}\right)
$$

where $\Omega_{I B}$ and $\Omega_{C G}$ consider the non-negative Lagrangian multipliers for $C 1$ and $C 2$, respectively. This can be obtained by taking the derivative of Equation (36) with respect to $d_{I B}$ and $d_{C G}$ to maximize the overall system throughput with interference control as follows:

$$
\frac{\partial L\left\{d_{I B}, d_{C G}, \Omega_{I B}, \Omega_{C G}\right\}}{\partial d_{I B}}=0
$$

Then,

$$
\Omega_{I B}=\frac{P_{C}\left|h_{C B}\right|^{2} \gamma_{C B}}{\left(I_{I B}+N_{o} B+P_{C}\left|h_{C B}\right|^{2} \gamma_{C B}\right)\left(I_{I B}+N_{o} B\right)}
$$

and

$$
\frac{\partial L\left\{d_{I B}, d_{C G}, \Omega_{I B}, \Omega_{C G}\right\}}{\partial d_{C G}}=0
$$

Then,

$$
\Omega_{C G}=\frac{P_{I}\left|h_{I G}\right|^{2} \gamma_{I G}}{\left(I_{C G}+N_{o} B+P_{I}\left|h_{I G}\right|^{2} \gamma_{I G}\right)\left(I_{C G}+N_{o} B\right)}
$$


Equations (37) and (38) show the values of $\Omega_{I B}$ and $\Omega_{C G}$ that satisfy the constraint of the optimization problem, respectively. By taking the derivative of Equation (36) with respect to $\Omega_{I B}$ and $\Omega_{C G}$,

$$
\begin{aligned}
& \frac{\partial L\left\{d_{I B}, d_{C G}, \Omega_{I B}, \Omega_{C G}\right\}}{\partial \Omega_{I B}}=0 \\
& \frac{\partial L\left\{d_{I B}, d_{C G}, \Omega_{I B}, \Omega_{C G}\right\}}{\partial \Omega_{C G}}=0
\end{aligned}
$$

we respectively conclude that:

$$
\begin{aligned}
& d_{I B}=\left(\frac{P_{I}\left|h_{I B}\right|^{2} \gamma_{o} S_{\text {INRthCB }}}{P_{C}\left|h_{C B}\right|^{2} \gamma_{C B}-S_{I N R t h C B} N_{o} B}\right)^{\frac{1}{\alpha}} \\
& d_{C G}=\left(\frac{P_{C}\left|h_{C G}\right|^{2} \gamma_{o} S_{\text {INRthIG }}}{P_{I}\left|h_{I G}\right|^{2} \gamma_{I G}-S_{\text {INRthIG }} N_{o} B}\right)^{\frac{1}{\alpha}}
\end{aligned}
$$

where $d_{I B}$ and $d_{C G}$ represent the optimum required distance between IoTD and BS and CUE and IoTG, respectively, to reduce the interference and enhance the system performance in terms of throughput.

The related results of the proposed approach will be shown in Section 4.

\section{Numerical Results and Discussion}

In this section, the performance of the proposed approach through MATLAB simulation is examined in terms of reliability, system outage probability, energy efficiency, and overall system throughput and is compared with previous related work $[15,18]$. The conditions for adapting the optimal required distance through the analysis of the results obtained are revealed. The network settings for the simulation are listed in Table 2. Figures 2 and 3 depict the optimum required distance between CUE and IoTG and IoTD and BS, respectively, which enhances the system performance and decreases the interference. Assume that $\mathrm{P}_{\mathrm{C}}$ and $\mathrm{P}_{\mathrm{I}}$ are $23 \mathrm{dBm}$ and $S I N R_{C B t h}$ and SINR $R_{I G \text { th }}$ are $10 \mathrm{~dB}$. As we can see from Figure 2, based on our assumption and scenario, the optimum required distance between IoTD and BS $\left(d_{I B}\right)$ must be approximately equal to 1.8 times the transmission distance between CUE and BS $\left(d_{C B}\right)$-for example, when $d_{C B}=300 \mathrm{~m}, d_{I B}$ must be at least $552 \mathrm{~m}$ to satisfy the system requirement, decrease interference, and enhance the system performance. The same result is obtained as shown in Figure 3 to enhance the IoT system performance; the optimum required distance between CUE and IoTG $\left(d_{C G}\right)$ must be approximately equal to 1.8 times the transmission distance between IoTD and IoTG $\left(d_{I G}\right)$-for example, when $d_{I G}=198 \mathrm{~m}, d_{C G}$ must be at least $354 \mathrm{~m}$. The obtained results can help in designing the IoT architecture and to determine the installation location for IoTD.

Table 2. Simulation parameters.

\begin{tabular}{cc}
\hline Parameters & Value \\
\hline$N_{o}$ & $-174 \mathrm{dBm}[27]$ \\
$B$ & $10 \mathrm{MHz}$ \\
$P_{o}$ & $20 \mathrm{dBm}$ \\
SINR $_{\text {thCB }}$ & $20 \mathrm{~dB}[31]$ \\
SINR $_{\text {thIG }}$ & $20 \mathrm{~dB}[31]$ \\
$P_{C}$ & $23 \mathrm{dBm}[31]$ \\
$P_{I}$ & $23 \mathrm{dBm}[31]$ \\
$F_{C}$ & $2 \mathrm{GHz}$ \\
$\mathrm{A}$ & 4 \\
$\gamma_{\mathrm{o}}$ & $10-1[31]$ \\
Number of CUEs (N) & $1-100$ \\
Number of IoTDs (K) & $1-100$ \\
\hline
\end{tabular}




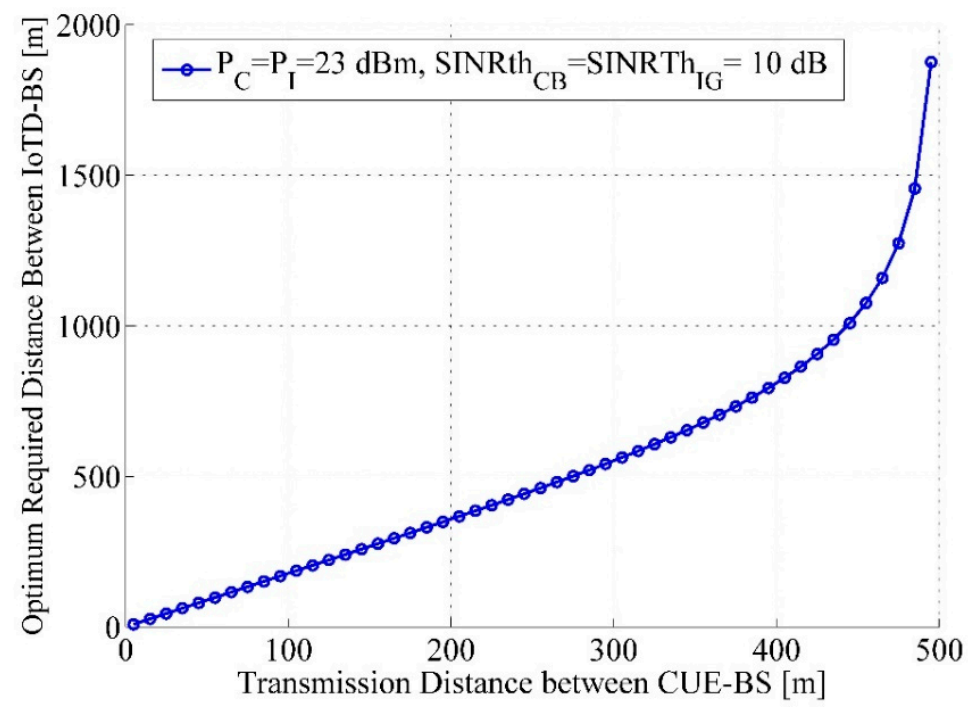

Figure 2. Transmission distance between cellular user and base station (CUE-BS) vs. optimum required distance between Internet of Things device and base station (IoTD-BS).

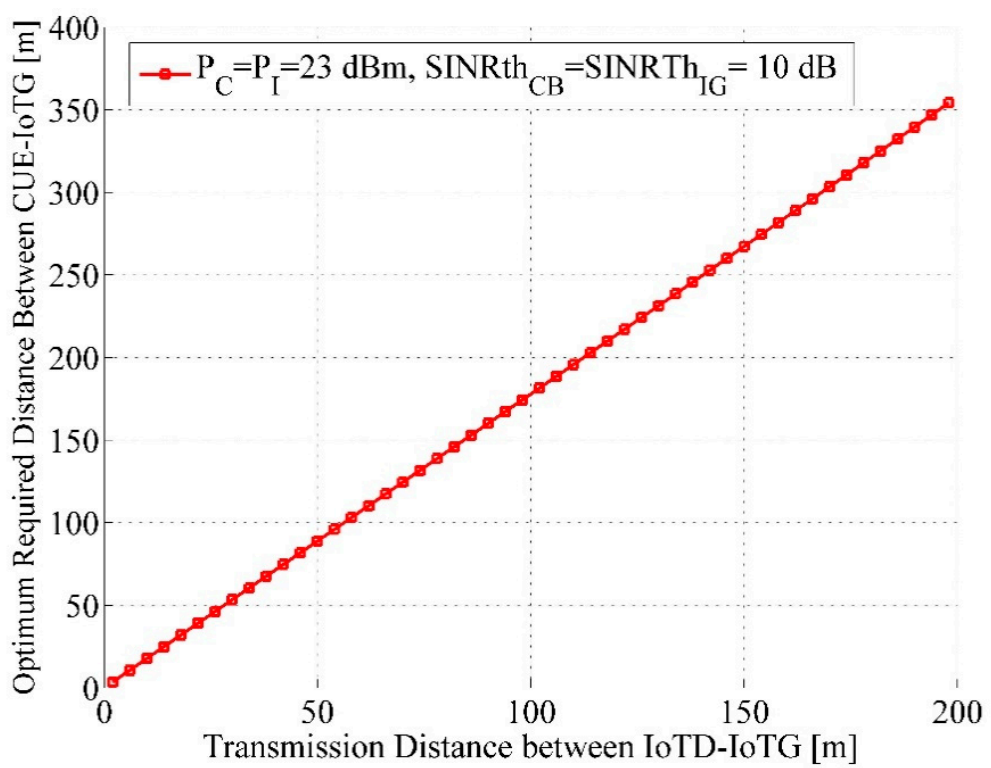

Figure 3. Transmission distance between IoTD and Internet of Things gateway (IoTG) vs. optimum required distance between CUE and IoTG.

The optimum required distance between IoTD and BS and CUE and IoTG is examined again for different scenarios but against $S I N R_{t h C B}$ and $S I N R_{t h I G}$, as shown in Figures 4 and 5. In Figure 4, we assume that $d_{C B}$ is 100,200 , and $300 \mathrm{~m}$ and $S I N R_{t h C B}$ varied from -10 to $20 \mathrm{~dB}$. As we can observe from Figure 4, each $S I N R_{t h C B}$ required a specific distance between IoTD and BS to satisfy the system requirements and enhance the system performance-for example, when $d_{C B}=200 \mathrm{~m}$ and $S I N R_{t h C B}=0 \mathrm{~dB}$, the optimum required distance between IoTD and BS is $200 \mathrm{~m}$, while when $d_{C B}=200 \mathrm{~m}$ and $\operatorname{SINR}_{t h C B}=15 \mathrm{~dB}$, the optimum required distance between IoTD and BS is $486 \mathrm{~m}$. In Figure 5, we assume that $d_{I G}$ is 40,80 , and $120 \mathrm{~m}$ and SINR $R_{t h I G}$ varied from -10 to $20 \mathrm{~dB}$. The optimum required distance between CUE and IoTG will be chosen based on the required SINR $R_{t h I G}$, as demonstrated in Figure 5-for example, when $d_{I G}=120 \mathrm{~m}$ and $S I N R_{t h I G}=0 \mathrm{~dB}$, the optimum required distance between CUE and IoTG is $200 \mathrm{~m}$; however, when $d_{I G}=120 \mathrm{~m}$ and $S I N R_{t h C B}=16 \mathrm{~dB}$, the optimum required distance between IoTD and BS is $301 \mathrm{~m}$. These two figures show that an adaptive smart system should 
be held based on the system requirements and environmental conditions to enhance the performance of the CUE and IoT networks.

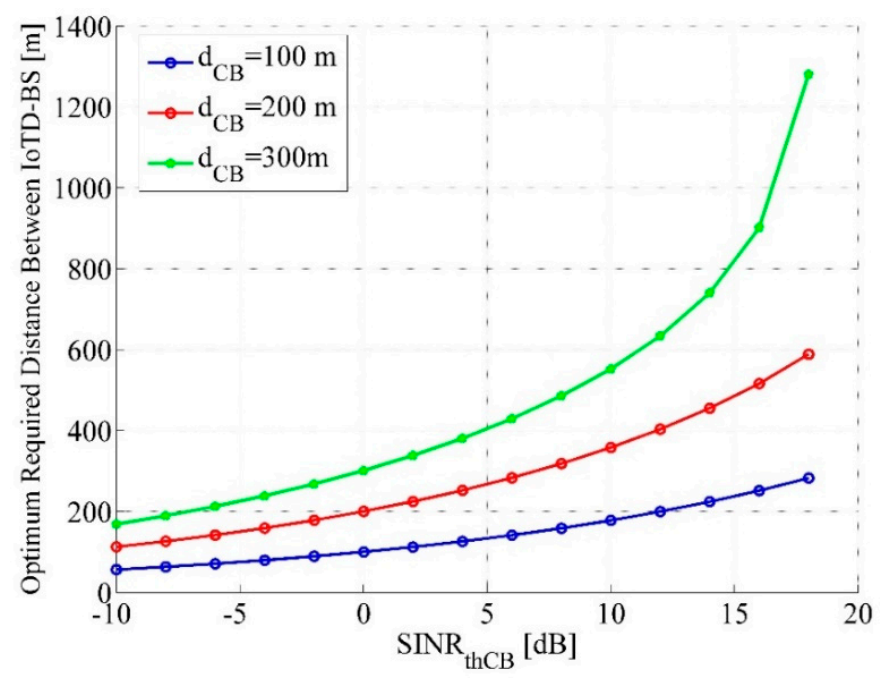

Figure 4. Signal-to-interference ratio at the CUE-BS link $\left(S I N R_{t h C B}\right)$ vs. optimum required distance between IoTD and BS.

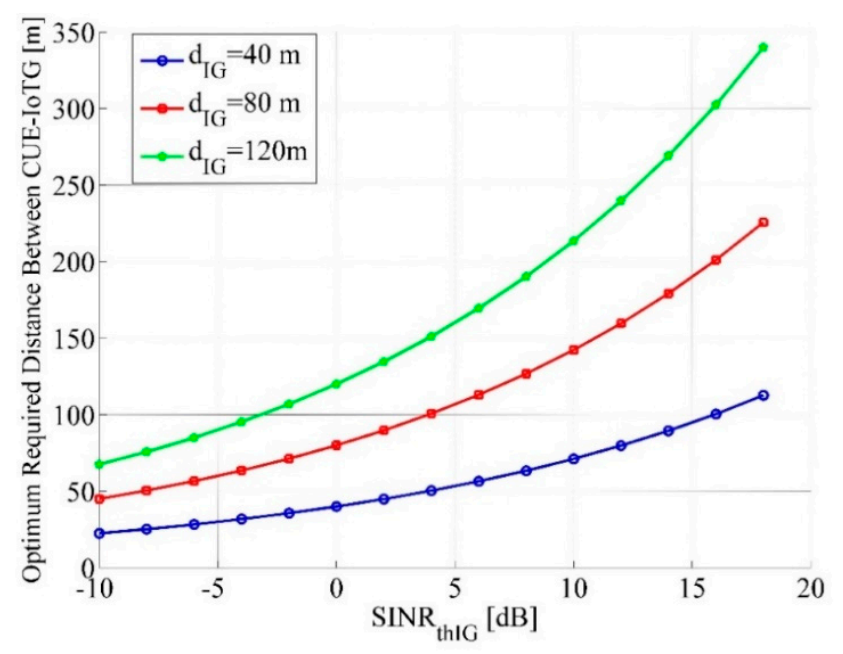

Figure 5. SINR $R_{\text {thIG }}$ vs. optimum required distance between IoTD and BS.

Figures 6 and 7 demonstrate the effect of CUE transmission power $\left(P_{C}\right)$ and IoTD transmission power $\left(P_{I}\right)$ on overall energy efficiency $(E E)$ and overall system throughput $\left(S_{t h}\right)$ for the proposed model and compare it with the model proposed in [15], assuming that $d_{C B}=200 \mathrm{~m}, d_{I G}=80 \mathrm{~m}$, and SINR $=S I N R_{\text {thIG }}=0 \mathrm{~dB}$. As shown in Figure 6, the increase in $P_{C}$ and $P_{I}$ leads to higher $E E$ in the proposed model as compared to the schemes previously proposed in [15], whereas after a certain threshold, the $E E$ reaches a peak and additional increase in maximum transmit power brings no enhancement in $E E$ but rather a decrease in $E E$, which means that for this scenario and based on the transmission distance and SINR between CUE and BS and IoTD and IoTG, the best CUE transmission power $\left(P_{C}\right)$ and IoTD transmission power $\left(P_{I}\right)$ is $6 \mathrm{dBm}$ for both to obtain the maximum $E E$. It is also noticed that the proposed optimization model outperforms the other previously proposed schemes and increases the overall EE. The same performance is obtained in Figure 7; the overall system throughput of the proposed model performs well comparing with the model proposed in [15]. It is also mentioned that the overall system throughput of the optimized proposed model for this transmission distance and 
SINR between CUE and BS and IoTD and IoTG increases when increasing $P_{C}$ and $P_{I}$, which means that maximizing the throughput of the system may lead to the decrease in the $E E$.

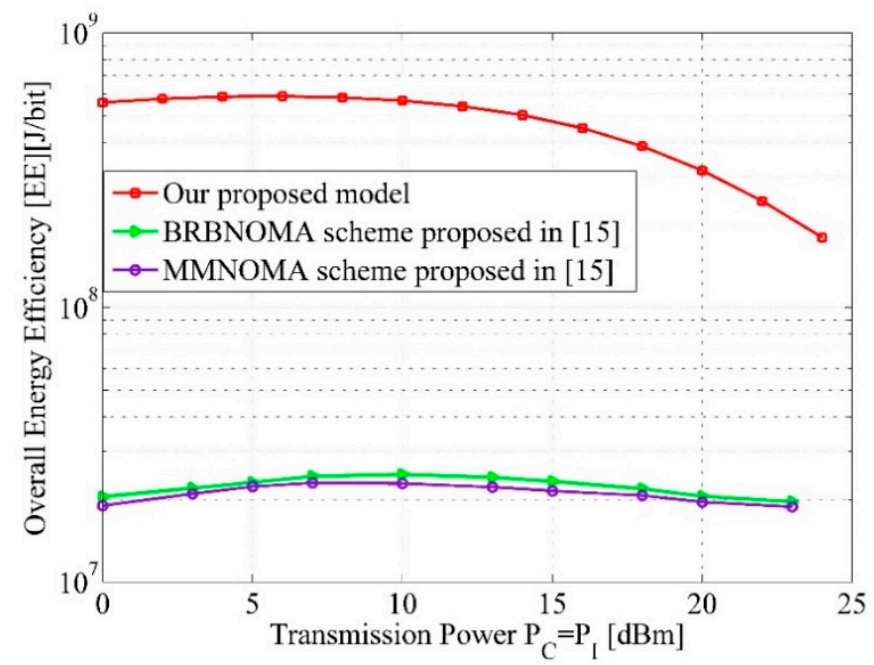

Figure 6. Transmission power $P_{C}$ and $P_{I}$ vs. overall energy efficiency $(E E)$.

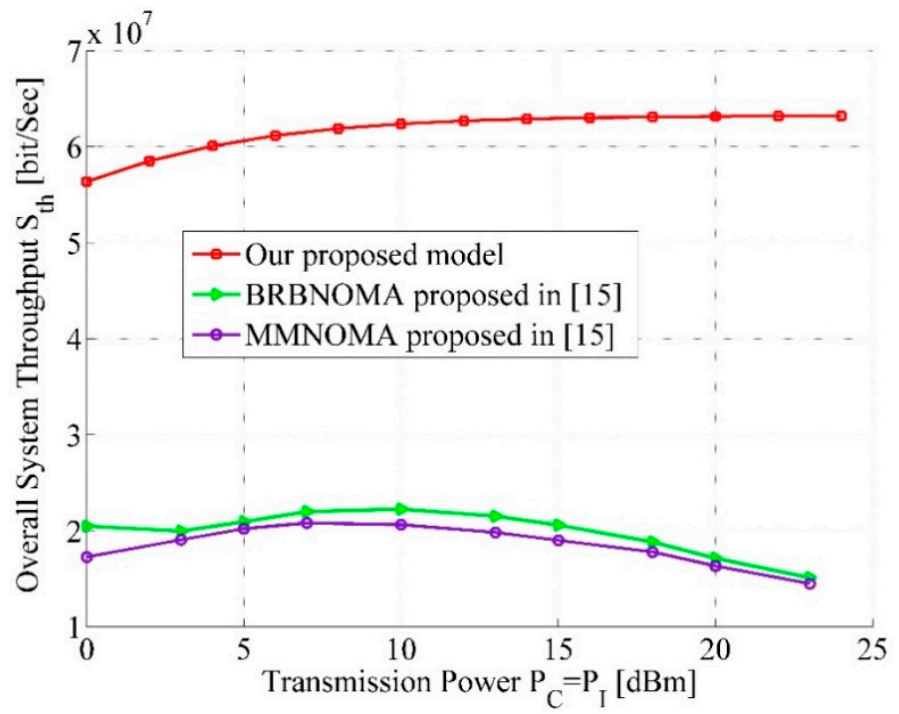

Figure 7. Transmission power $P_{C}$ and $P_{I}$ vs. overall system throughput $\left(S_{t h}\right)$.

We can conclude from the results obtained in Figures 6 and 7 that each transmission distance and each SINR between CUE and BS and IoTD and IoTG leads to determining the required transmission power of CUE and IoTD to achieve the maximum EE with the best overall system throughput. Furthermore, these two figures demonstrate the effectiveness and the accuracy of the proposed model when comparing it with the other model in [15].

To better clarify the results obtained in Figures 6 and 7, we plotted Figure 8 to present the EE versus the overall system throughput. For a low throughput, EE increases with the throughput. However, $E E$ decreases with throughput when it is larger. It means that there is a tradeoff between the $E E$ and throughput, namely, increasing the throughput may decrease the $E E$. In practical systems, for obtaining the maximum EE with the best system throughput, the two performances can be jointly considered in order to attain the required desirable tradeoff between the two metrics under different conditions. The system performance is evaluated in terms of reliability and overall system outage probability versus the number of CUEs and IoTDs, where $P_{C}=P_{I}=23 \mathrm{dBm}$, and SINR $\operatorname{shCB}_{\text {C }}=S I N R_{\text {thIG }}=20 \mathrm{~dB}$. Figure 9 demonstrates that increasing the number of CUEs and IoTDs leads to higher reliability for the 
proposed model and the model proposed in [18], which means that both models aim to enhance the system reliability using different techniques regardless of the number of transmissions. The technique presented in [18] was based on determining a minimum number of channels to be sensed by each IoT node in a multiband approach to enhance system reliability and increase EE, while our proposed model is based on adapting the optimum required distance between CUE and IoTG and the system performance is evaluated in terms of reliability and overall system outage probability versus the number of CUEs and IoTD, where $P_{C}=P_{I}=23 \mathrm{dBm}$, and $\operatorname{SINR} R_{t h C B}=S I N R_{\text {thIG }}=20 \mathrm{~dB}$.

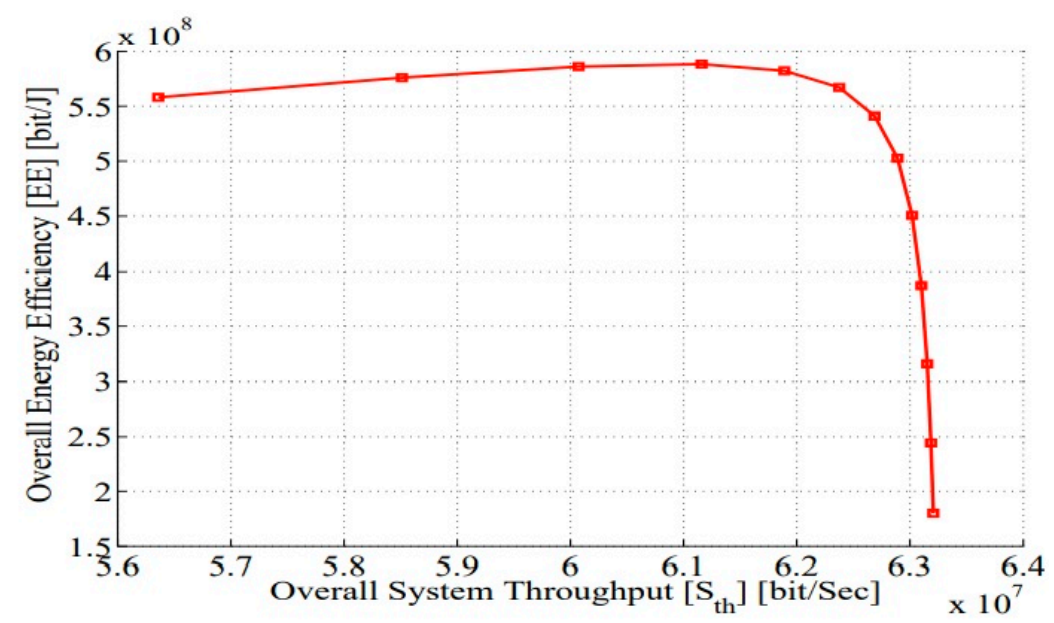

Figure 8. Overall system throughput $\left(S_{t h}\right)$ vs. overall energy efficiency $(E E)$.

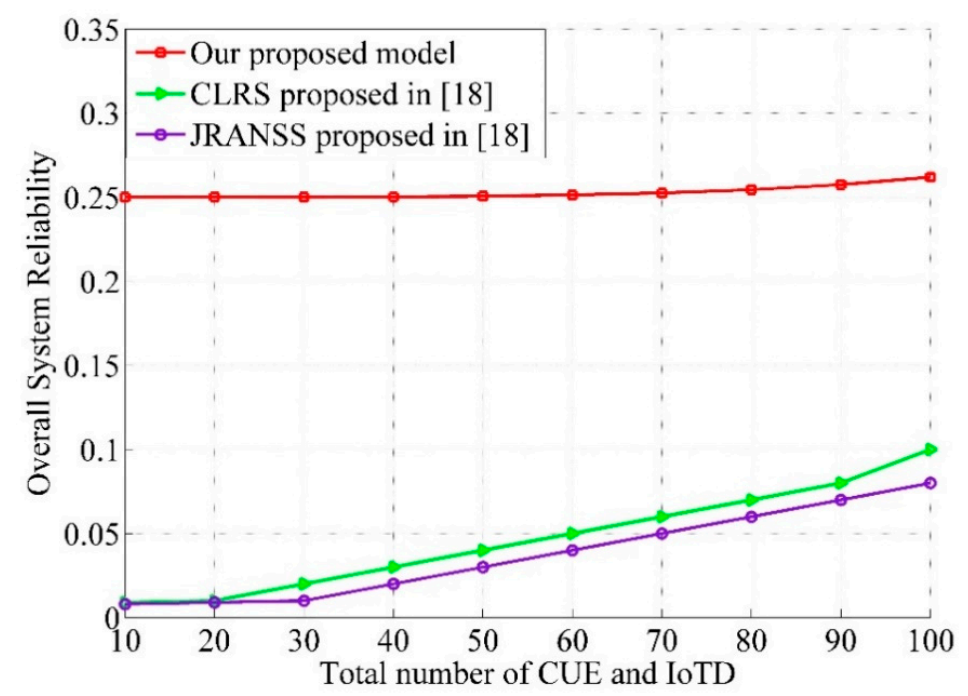

Figure 9. Total number of CUEs and IoTDs vs. overall system reliability.

The system performance is evaluated in terms of reliability and overall system outage probability versus the number of CUEs and IoTDs, where $P_{C}=P_{I}=23 \mathrm{dBm}$, and $\operatorname{SINR} R_{t h C B}=S I N R_{\text {thIG }}=20 \mathrm{~dB}$. Figure 9 demonstrates that increasing the number of CUEs and IoTDs leads to higher reliability for the proposed model and the model proposed in [18], which means that both models aim to enhance the system reliability using different techniques, whatever the number of transmissions is. The technique presented in [18] was based on determining a minimum number of channels to be sensed by each IoT node in a multiband approach to enhance system reliability and increase $E E$, while our proposed model is based on adapting the optimum required distance between CUE and IoTG and IoTD and BS based on the transmission distance between CUE and BS and IoTD and IoTG to decrease the interference, enhance system reliability, and increase $E E$ and system throughput. It is also mentioned that our proposed framework outperforms the model proposed in [18]. The same performance is obtained 
when evaluating the system performance in terms of overall system outage probability, as shown in Figure 10. Figure 10 depicts that increasing the number of sender nodes decreases the system outage probability for both models, and our proposed model has better performance than the model proposed in [18]. This result is due to the high link reliability for any CUE-BS and IoTD-IoTG, which also reflects the results obtained in Figure 9.

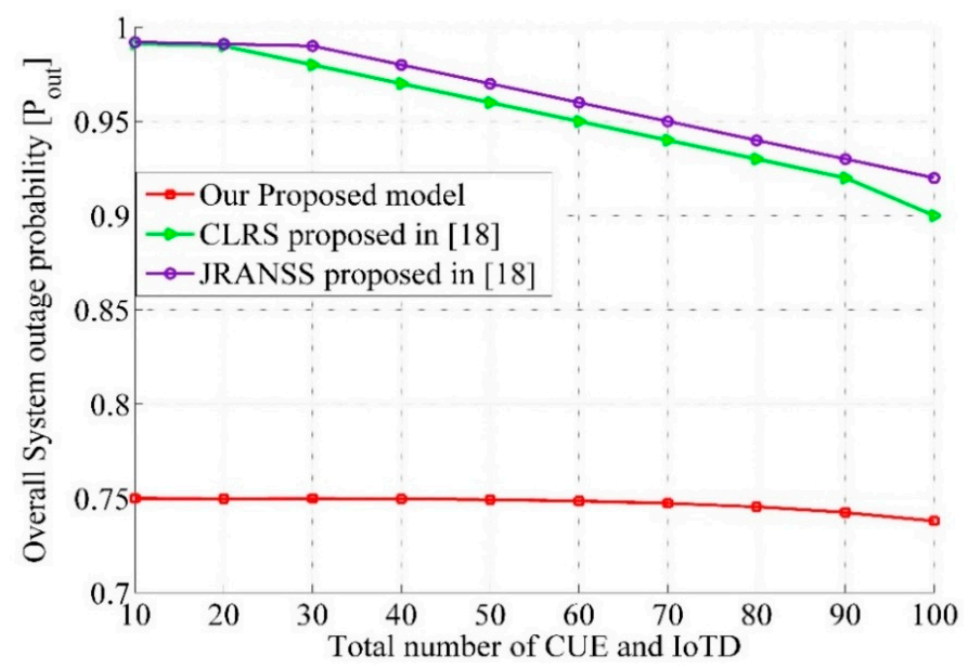

Figure 10. Total number of CUEs and IoTDs vs. overall system outage probability $\left(P_{\text {out }}\right)$.

Finally, we examine the overall system throughput and energy efficiency for the different number of CUEs and IoTDs, assuming $P_{C}=P_{I}=23 \mathrm{dBm}$ and SINR $R_{t h C B}=S I N R_{t h I G}=20 \mathrm{~dB}$, and the distances between the CUE and BS and IoTD and IoTG links are randomly chosen and vary between 10 and $500 \mathrm{~m}$. Figure 11 depicts the overall system throughput of the proposed model and the model proposed in [18] versus the number of CUEs and IoTDs. As it is shown, the overall system throughput increases when the number of CUEs and IoTDs increases for both models, as increasing the number of sender nodes increases the number of transmitted packets, which increases the overall system throughput. This also reflects the result obtained in Figure 9; higher reliability means higher system throughput. It is worth mentioning that the proposed model has a much higher overall system throughput than the model proposed in [18]; this is due to the suggested proposed model being based on adapting the distances between IoTD and BS and CUE and IoTG, which decreases the interference between IoTD and BS and CUE and IoTG and thus improves the system performance.

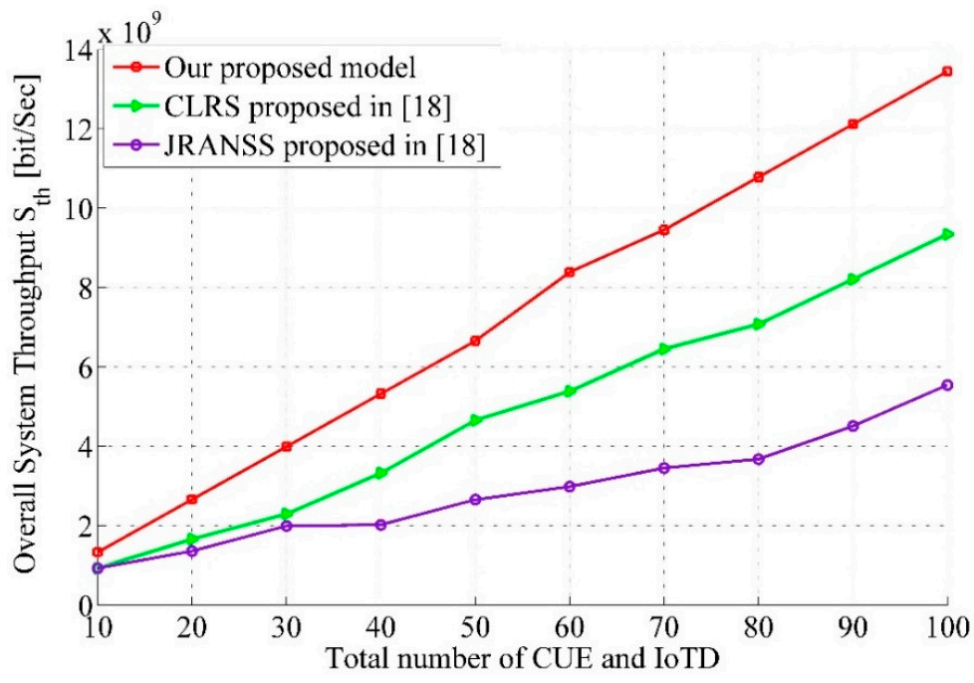

Figure 11. Total number of CUEs and IoTDs vs. overall system throughput $\left(S_{t h}\right)$. 
Figure 12 depicts the energy efficiency of the proposed model and the model proposed in [18] by counting on all possible transmission distances between CUE-BS and IoTD-IoTG links. As illustrated in Figure 12 the proposed model in [18] has lower energy efficiency than the proposed model, additionally, it shows that for both models EE gradually increases when the number of CUE and IoTD increases. As mentioned previously, increasing the number of sender nodes increases the number of transmitted packets which increases the overall system throughput and then increases $E E$. The result obtained in this figure and Figures 9 and 11 show the effectiveness and the accuracy of the proposed model compared with other previously proposed models.

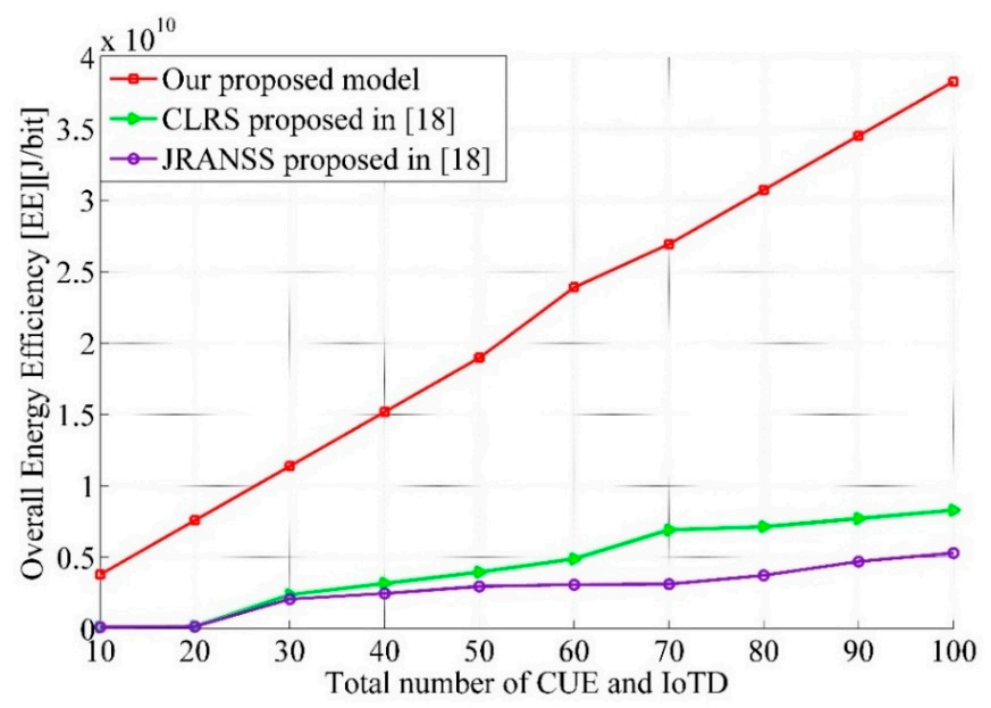

Figure 12. Total number of CUEs and IoTDs vs. overall energy efficiency $(E E)$.

A summarized analysis for the performance of the proposed model can be given based on the above discussions and the results presented in Figures 2-12. Firstly, based on our analysis, there are several factors affecting system throughput, energy efficiency, reliability, and outage probability. The idea of the proposed approach is an adaptive way to adjust the distance between IoTD-BS $\left(d_{I B}\right)$ and CUE-IoTG $\left(d_{C G}\right)$, which decreases the interference that occurs at BS and IoTG and affects the system performance. $\left(d_{I B}\right)$ and $\left(d_{C G}\right)$ will be adapted based on different environmental conditions such as path loss exponent, $\left(d_{C B}\right)$ and $\left(d_{I G}\right)$, and the required QoS to enhance the system performance.

Secondly, in the evaluation of the QoS performance of the proposed model, we demonstrate a clear advantage of the proposed approach over the other models. Specifically, an important performance measure for QoS is reliability and also system outage probability, which is affected by the transmission power, as decreasing the transmission power affects the system outage probability; on the other hand, if the transmission power increases, the interference at the BS and IoTG increases due to the different number of CUEs and IoTDs. As a result of the overall impact of this factor on throughput and energy efficiency, a desirable tradeoff between them must be jointly evaluated to show improvement in the system performance to maintain the QoS required.

Clearly, based on the requirement of a particular application, to achieve the best system performance in terms of throughput, energy efficiency, outage probability, and system reliability, proper distance between CUE-IoTG and IoTD-BS should be adapted for the given transmission conditions, such as overall distance, $d_{C B}, d_{I G}$, channel quality in terms of $\alpha$, and the transmission power of CUE $\left(P_{C}\right)$ and IoTD $\left(P_{I}\right)$. Our work provides effective guidance for deciding when and how the CUEs and IoTDs should be communicating and be placed. For achieving high throughput and energy efficiency as the main QoS requirement, the proposed approach is recommended for the given transmission conditions. Therefore, based on our results, the enhancement of cellular and IoT network performances can be achieved by adaptively choosing the appropriate distance between CUE-IoTG and IoTD-BS under different network and transmission conditions. 


\section{Conclusions}

A new framework called the interference control model was proposed to control interference among IoTG and BS and is based on using the Lagrange optimization technique to reduce interference and improve the QoS by maximizing the energy efficiency and reliability of IoT and cellular networks in fifth-generation (5G) systems. Based on the system throughput, energy efficiency, and reliability, we have shown that the proposed model can exhibit the best performance under a certain environmental condition compared with other proposed models. The optimal distance between CUE-IoTG and IoTD-BS can be identified by judging the distance between CUE-BS and IoTD-IoTG links to decrease the interference in both links. The results presented in this paper can be used to form an adaptive interference control to achieve the best system performance for cellular and IoT networks. In this way, we can attain the highest throughput and energy efficiency with suitable system reliability.

Author Contributions: Conceptualization, R.A.O.; Methodology, A.I.Z. and R.A.O.; Software, R.A.O.; Writing-original draft preparation, R.A.O.; Writing—review and editing, A.I.Z. All authors have read and agreed to the published version of the manuscript.

Funding: This research received no external funding.

Conflicts of Interest: The authors declare no conflict of interest.

\section{References}

1. Wang, H.; Xiong, D.; Wang, P.; Liu, Y. A lightweight xmpp publish/subscribe scheme for resource-constrained iot devices. IEEE Access 2017, 5, 16393-16405. [CrossRef]

2. Liu, X.; Ansari, N. Green relay assisted d2d communications with dual battery for iot. In Proceedings of the 2016 IEEE Global Communications Conference (GLOBECOM), Washington, DC, USA, 4-8 December 2016; pp. 1-6.

3. Chen, S.; Xu, H.; Liu, D.; Hu, B.; Wang, H. A vision of iot: Applications, challenges, and opportunities with china perspective. IEEE Internet Things J. 2014, 1, 349-359. [CrossRef]

4. Luong, N.C.; Hoang, D.T.; Wang, P.; Niyato, D.; Kim, D.I.; Han, Z. Data collection and wireless communication in internet of things (iot) using economic analysis and pricing models: A survey. IEEE Commun. Surv. Tutor. 2016, 18, 2546-2590. [CrossRef]

5. Giuliano, R.; Mazzenga, F.; Neri, A.; Vegni, A.M. Security access protocols in iot capillary networks. IEEE Internet Things J. 2017, 4, 645-657. [CrossRef]

6. Zhao, M.; Ho, I.W.-H.; Chong, P.H.J. An energy-efficient region-based rpl routing protocol for low-power and lossy networks. IEEE Internet Things J. 2016, 3, 1319-1333. [CrossRef]

7. Dawy, Z.; Saad, W.; Ghosh, A.; Andrews, J.G.; Yaacoub, E. Toward massive machine type cellular communications. IEEE Wirel. Commun. 2017, 24, 120-128. [CrossRef]

8. Agiwal, M.; Roy, A.; Saxena, N. Next generation $5 \mathrm{~g}$ wireless networks: A comprehensive survey. IEEE Commun. Surv. Tutor. 2016, 18, 1617-1655. [CrossRef]

9. Li, S.; Xu, L.D.; Zhao, S. 5g internet of things: A survey. J. Ind. Inf. Integr. 2018, 10, 1-9. [CrossRef]

10. Vogler, M.; Schleicher, J.; Inzinger, C.; Dustdar, S. Optimizing elastic iot application deployments. IEEE Trans. Serv. Comput. 2016, 11, 879-892. [CrossRef]

11. Palattella, M.R.; Dohler, M.; Grieco, A.; Rizzo, G.; Torsner, J.; Engel, T.; Ladid, L. Internet of things in the $5 \mathrm{~g}$ era: Enablers, architecture, and business models. IEEE J. Sel. Areas Commun. 2016, 34, 510-527. [CrossRef]

12. Akpakwu, G.A.; Silva, B.J.; Hancke, G.P.; Abu-Mahfouz, A.M. A survey on $5 \mathrm{~g}$ networks for the internet of things: Communication technologies and challenges. IEEE Access 2018, 6, 3619-3647. [CrossRef]

13. Sun, M.; Shi, Z.; Chen, S.; Zhou, Z.; Duan, Y. Energy-efficient composition of configurable internet of things services. IEEE Access 2017, 5, 25609-25622. [CrossRef]

14. Khanouche, M.E.; Amirat, Y.; Chibani, A.; Kerkar, M.; Yachir, A. Energy-centered and qosaware services selection for internet of things. IEEE Trans. Autom. Sci. Eng. 2016, 13, 1256-1269. [CrossRef]

15. Chinnadurai, S.; Yoon, D.D. Energy efficient mimo-noma hen with iot for wireless communication systems. In Proceedings of the 2018 International Conference on Information and Communication Technology Convergence (ICTC), Jeju Island, Korea, 17-19 October 2018; pp. 856-859. 
16. Lv, T.; Lin, Z.; Huang, P.; Zeng, J. Optimization of the energy-efficient relay-based massive iot network. IEEE Internet Things J. 2018, 5, 3043-3058. [CrossRef]

17. Zhang, L.; Liang, Y.-C. Average throughput analysis and optimization in cooperative iot networks with short packet communication. IEEE Trans. Veh. Technol. 2018, 67, 11549-11562. [CrossRef]

18. Ejaz, W.; Ibnkahla, M. Multiband spectrum sensing and resource allocation for iot in cognitive $5 \mathrm{~g}$ networks. IEEE Internet Things J. 2017, 5, 150-163. [CrossRef]

19. Chen, J.; Zhang, L.; Liang, Y.-C.; Kang, X.; Zhang, R. Resource allocation for wireless-powered iot networks with short packet communication. IEEE Trans. Wirel. Commun. 2019, 18, 1447-1461. [CrossRef]

20. Biason, A.; Pielli, C.; Rossi, M.; Zanella, A.; Zordan, D.; Kelly, M.; Zorzi, M. Ec-centric: An energy-and context-centric perspective on iot systems and protocol design. IEEE Access 2017, 5, 6894-6908. [CrossRef]

21. Liu, X.; Ansari, N. Green relay assisted d2d communications with dual batteries in heterogeneous cellular networks for iot. IEEE Internet Things J. 2017, 4, 1707-1715. [CrossRef]

22. Song, L.; Chai, K.K.; Chen, Y.; Schormans, J.; Loo, J.; Vinel, A. Qos-aware energy-efficient cooperative scheme for cluster-based iot systems. IEEE Syst. J. 2017, 11, 1447-1455. [CrossRef]

23. Ercan, A.Ö.; Sunay, M.O.; Akyildiz, I.F. Rf energy harvesting and transfer for spectrum sharing cellular iot communications in 5g systems. IEEE Trans. Mob. Comput. 2018, 17, 1680-1694. [CrossRef]

24. Yang, Z.; Xu, W.; Pan, Y.; Pan, C.; Chen, M. Energy efficient resource allocation in machineto-machine communications with multiple access and energy harvesting for iot. IEEE Internet Things J. 2018, 5, 229-245. [CrossRef]

25. Li, J.; Xiong, K.; Cao, J.; Yang, X.; Liu, T. Energy Efficiency in RF Energy Harvesting-Powered Distributed Antenna Systems for the Internet of Things. Sensors 2020, 20, 4631. [CrossRef] [PubMed]

26. Mostafa, B.; Benslimane, A.A.; Saleh, M.; Kassem, S.; Molnar, M. An energy-efficient multiobjective scheduling model for monitoring in internet of things. IEEE Internet Things J. 2018, 5, 1727-1738. [CrossRef]

27. Huang, Y.; Liu, M.; Liu, Y. Energy-efficient swipt in iot distributed antenna systems. IEEE Internet Things J. 2018, 5, 2646-2656. [CrossRef]

28. Lee, H.; Jang, H.S.; Jung, B.C. Improving Energy Efficiency Fairness of Wireless Networks: A Deep Learning Approach. Energies 2019, 12, 4300. [CrossRef]

29. Jiang, H.; Xiao, Z.; Li, Z.; Xu, J.; Zeng, F.; Wang, D. An Energy-Efficient Framework for Internet of Things Underlaying Heterogeneous Small Cell Networks. IEEE Trans. Mob. Comput. 2020. [CrossRef]

30. Fu, S.; Su, Z.; Jia, Y.; Zhou, H.; Jin, Y.; Ren, J.; Wu, B.; Huq, K.M.S. Interference cooperation via distributed game in 5 g networks. IEEE Internet Things J. 2017, 6, 310-320. [CrossRef]

31. Chae, S.H.; Jeon, S.-W.; Jeong, C. Efficient resource allocation for iot cellular networks in the presence of inter-band interference. IEEE Trans. Commun. 2019, 67, 4299-4308. [CrossRef]

32. Zhang, L.; Ijaz, A.A.; Xiao, P.; Molu, M.M.; Tafazolli, R. Filtered ofdm systems, algorithms, and performance analysis for $5 \mathrm{~g}$ and beyond. IEEE Trans. Commun. 2018, 66, 1205-1218. [CrossRef]

33. Liu, S.; Yang, F.; Song, J.; Han, Z. Block sparse bayesian learning-based nb-iot interference elimination in lte-advanced systems. IEEE Trans. Commun. 2017, 65, 4559-4571. [CrossRef]

34. ElHalawany, B.M.; Ruby, R.; Wu, K. D2d communication for enabling internet-of-things: Outage probability analysis. IEEE Trans. Veh. Technol. 2019, 68, 2332-2345. [CrossRef]

35. Fan, B.; Tian, H.; Jiang, L.; Vasilakos, A.V. A social-aware virtual mac protocol for energy efficient d2d communications underlying heterogeneous cellular networks. IEEE Trans. Veh. Technol. 2018, 67, 8372-8385. [CrossRef]

36. Zhang, Q.; Zhang, L.; Liang, Y.-C.; Kam, P.-Y. Backscatter-noma: A symbiotic system of cellular and internet-of-things networks. IEEE Access 2019, 7, 20000-20013. [CrossRef]

Publisher's Note: MDPI stays neutral with regard to jurisdictional claims in published maps and institutional affiliations. 